\title{
BMJ Open Effects of thyroid-stimulating hormone suppression after thyroidectomy for thyroid cancer on bone mineral density in postmenopausal women: a systematic review and meta-analysis
}

\author{
Donghee Kwak, ${ }^{1,2}$ Jane Ha (D) , ${ }^{3,4}$ Yousun Won, ${ }^{5}$ Yeongkeun Kwon, ${ }^{4,6}$ \\ Sungsoo Park ${ }^{4,6}$
}

To cite: Kwak D, Ha J, Won Y, et al. Effects of thyroid-stimulating hormone suppression after thyroidectomy for thyroid cancer on bone mineral density in postmenopausal women: a systematic review and meta-analysis. BMJ Open 2021;11:e043007. doi:10.1136/ bmjopen-2020-043007

- Prepublication history and additional supplemental material for this paper are available online. To view these files, please visit the journal online (http://dx.doi.org/10.1136/ bmjopen-2020-043007).

DK and JH contributed equally.

Received 21 July 2020 Revised 01 May 2021 Accepted 04 May 2021

Check for updates

(C) Author(s) (or their employer(s)) 2021. Re-use permitted under CC BY-NC. No commercial re-use. See rights and permissions. Published by BMJ.

For numbered affiliations see end of article.

Correspondence to Professor Yeongkeun Kwon; kukwon@korea.ac.kr

\section{ABSTRACT}

Objectives We assessed thyroid-stimulating hormone (TSH) suppression effects on bone mineral density (BMD) in postmenopausal women who underwent thyroidectomy.

Data sources PubMed, EMBASE, Cochrane Library, Web of Science and SCOPUS were searched from inception to 24 February 2021.

Study selection Case-control studies were included. Data extraction and synthesis Two authors independently reviewed the studies, extracted the data and performed meta-analysis of eligible studies.

Research design and methods Studies evaluating BMD in postmenopausal women with thyroid cancer who had thyroidectomy and levothyroxine therapy were included. Differences in BMD were presented as standardised mean differences (SMDs). Meta-analyses were conducted using a random-effects model.

Results Analysis of 16 case-control studies (426 patients and 701 controls without thyroid cancer) showed that stringent TSH suppression (TSH $<0.10 \mathrm{mlU} / \mathrm{L}$ ) after thyroidectomy had deleterious effects on the BMD of the lumbar spine in postmenopausal women compared with controls (SMD $-0.55 ; 95 \% \mathrm{Cl}-0.99$ to -0.10 ; $\left.\mathrm{I}^{2}=75.8 \%\right)$. There was no significant difference in patients with moderate TSH suppression (TSH 0.10-0.49 mlU/L). TSH suppression in postmenopausal women was not significantly associated with lower femoral neck BMD. Subgroup analysis of the lumbar spine showed that the association between stringent TSH suppression and lower BMD was consistent among studies with $>10$ years of follow-up (SMD $-0.32 ; 95 \% \mathrm{Cl}-0.50$ to -0.14 ). Subgroup analysis of the femoral neck showed that total thyroidectomy was related to detrimental effects on the BMD of the femoral neck (SMD $-0.60 ; 95 \% \mathrm{Cl}-0.89$ to $\left.-0.31 ; I^{2}=90.4 \%\right)$, but near-total thyroidectomy was not (SMD $0.00 ; 95 \% \mathrm{Cl}-0.30$ to $0.30 ; \mathrm{l}^{2}=55.6 \%$ ).

Conclusions Stringent TSH suppression had deleterious effects on the BMD of the lumbar spine after thyroidectomy in postmenopausal women. Further studies are needed to determine whether stringent TSH suppression after thyroidectomy increases the fracture risk.
Strengths and limitations of this study

- This is the first systematic review and meta-analysis to qualitatively collate and assess the results of studies that investigated the effect of thyroid-stimulating hormone suppression after thyroidectomy for thyroid cancer on bone mineral density in postmenopausal women.

- We investigated the association between thyroidstimulating hormone suppression and lumbar spine or femoral neck bone mineral density in different subgroups (follow-up duration, surgical extent, ethnicity and body mass index).

- A possible limitation is that only a few prospective studies were included, and the effects of notable confounding factors and possible fluctuations in the postoperative thyroid-stimulating hormone levels were not reflected in the analysis.

\section{INTRODUCTION}

Thyroid-stimulating hormone (TSH) levels are suppressed after thyroidectomy for thyroid $^{\text {cancer. }}{ }^{1-3}$ It is necessary to assess the risk-benefit and adverse effects of $\mathrm{TSH}$ suppression because patients with thyroid cancer have a favourable prognosis. ${ }^{4}$ To prevent cancer recurrence and reduce cancer-related mortality, levothyroxine is used to achieve TSH suppression, ${ }^{5}$ as it inhibits the production of circulating TSH, a factor known to stimulate the proliferation of thyrocytes. ${ }^{6}$ However, supraphysiological doses of exogenous levothyroxine may cause side effects, particularly regarding bone health. ${ }^{2} 7$ Endogenous hyperthyroidism increases the risk of osteoporosis and osteoporotic fractures. ${ }^{89}$ However, the effects of TSH suppression due to levothyroxine administration after thyroidectomy are controversial. ${ }^{10}$ Some studies reported a lack of deleterious 
effects on the bone mineral density (BMD) of long-term thyroxine suppressive therapy for differentiated thyroid carcinoma. ${ }^{11-14}$ In contrast, other studies reported that thyroxine suppressive therapy had a detrimental effect on BMD. ${ }^{1516}$

Although different levels and durations of TSH suppression are recommended post-thyroidectomy depending on the risk of thyroid cancer recurrence, ${ }^{11718}$ the impact of varying levels of TSH suppression on bone health is unclear. Several studies have evaluated the association between TSH suppression levels and fractures. ${ }^{8} 1920$ One of them reported that women with a low TSH level $(<0.1 \mathrm{mU} / \mathrm{L})$ had a threefold increased risk for hip fractures and a fourfold increased risk for vertebral fractures compared with women who had normal TSH levels. ${ }^{20}$ However, this study focused on the risk of fracture rather than BMD in women with low serum TSH levels. Several literature reviews have reported that suppressed TSH levels lead to osteoporosis and decreased BMD after thyroidectomy for thyroid cancer in postmenopausal women. ${ }^{21-26}$ However, no further studies have investigated the effects of varying TSH levels on BMD.

In this study, we performed a systematic review and meta-analysis of the current literature to investigate the effects of different levels of TSH suppression after thyroidectomy on postoperative BMD in postmenopausal women with thyroid cancer.

\section{MATERIALS AND METHODS}

An expert panel was formed to define the scope of the meta-analysis, literature search, eligibility criteria and statistical methods according to the preferred reporting items for systematic reviews and meta-analysis guidelines. ${ }^{27}$ This systematic review and meta-analysis protocol was registered in the PROSPERO database (registration number, CRD42018104282). At the time of initial registration of PROSPERO, patients who underwent thyroidectomy due to any thyroid disease were registered. However, there are few studies on BMD after thyroidectomy in typical hyperthyroidism diseases such as Graves' disease, toxic multinodular goitre and toxic adenoma, excluding cancer, so our study was limited to only patients with thyroid cancer.

\section{Patient and public involvement statement}

This meta-analysis was based on study-level data, and no individual-level data were involved in the study or in defining the research question or outcome measures.

\section{Literature search}

A trained librarian searched the PubMed, EMBASE, Cochrane Library, Web of Science and SCOPUS databases to identify relevant studies published before 24 February 2021 (online supplemental table 1). The following search terms, adapted to each database, were used: "thyroidectomy", "levothyroxine", "thyroid stimulating hormone", "bone density", "osteoporosis" and "bone fractures". We also included studies reported in earlier systematic reviews, ${ }^{21-24} 26$ and those identified from the bibliography of the included articles.

\section{Eligibility criteria and study selection}

The inclusion criteria were as follows: (1) all participants underwent thyroidectomy for thyroid cancer, followed by the administration of levothyroxine; (2) availability of BMD data, irrespective of whether this was the primary end point; (3) availability of data on the lumbar spine and femoral neck; (4) comparison of postoperative outcomes between the study and control groups (matched healthy postmenopausal women were enrolled) and (5) the most recent or informative publication data, in the case of multiple publications from the same population. The exclusion criteria were as follows: (1) only abstract available; (2) case reports, reviews and editorials; (3) studies with paediatric or pregnant populations; (4) publication language other than English and (5) missing measures of variability (SDs, SEs or IQRs) when effect estimates were presented.

Two authors (DK and YK) independently assessed the eligibility of all studies identified through an electronic literature search based on the titles and abstracts. Eligible articles with full texts were further evaluated for inclusion. Disagreements were resolved by consensus.

\section{Data extraction and risk-of-bias assessment}

A standard template suggested by Cochrane was adapted and applied for data extraction. ${ }^{28}$ Two authors (DK and $\mathrm{JH}$ ) independently extracted the data, including the first author's name; year of publication; study location; study design; age, body mass index (BMI), ethnicity, number of the study participants; aetiology; extent of thyroidectomy; location and value of the BMD measurement; follow-up duration after thyroidectomy; levothyroxine dose; preoperative and postoperative laboratory results including $\mathrm{TSH}$, free thyroxine, parathyroid hormone and calcium levels; medications used by the study participants and comorbidities.

The quality of the included studies was assessed using the Newcastle-Ottawa Quality Assessment Scale. ${ }^{29}$ This assesses the quality of non-randomised studies according to three variables: selection of the study population, comparability and exposure factor.

\section{Data synthesis and analysis}

Each outcome was presented with estimates calculated as standardised mean differences (SMDs). Assuming that the baseline values of patients and controls without thyroid cancer were balanced and the confounders were controlled by the study design, we compared the measurements at the end of the follow-up period. The SMDs were coded so that positive values represented a higher BMD in the thyroidectomy group than in the control group, with $0.2,0.5$ and 0.8 , corresponding to small, moderate and large effects, respectively. ${ }^{30}$ We estimated the pooled SMDs and 95\% CIs using the random-effects model 
(DerSimonian and Laird method). Statistical significance was set at a two-tailed $p$ value of $<0.05$. We assessed heterogeneity using the Mantel-Haenszel model and $\mathrm{I}^{2}$ values (the percentage of variance in the pooled estimate due to between-study differences), with $\mathrm{I}^{2}$ thresholds of $\leq 25 \%$ (low), 26\%-50\% (moderate) and $>50 \%$ (high).

Subgroup analyses were performed considering the TSH level (categorised as stringent suppression, $<0.10 \mathrm{mIU} / \mathrm{L}$; moderate suppression, $0.10-0.49 \mathrm{mIU} / \mathrm{L}$ and lower normal, $0.50-2.0 \mathrm{mIU} / \mathrm{L}$ ). Other investigated factors included the follow-up duration, baseline age, baseline BMI, ethnicity and extent of thyroidectomy (total vs near-total). Publication bias was assessed based on funnel plot asymmetry using Begg's and Egger's tests at a $\mathrm{p}$ value of $<0.1$. Stata V.13.1 software (StataCorp, College Station, Texas, USA) was used for all statistical analyses.

\section{RESULTS}

\section{Studies included in the meta-analysis}

A systematic literature search retrieved a total of 3063 studies (online supplemental figure 1). We included two studies from the bibliography of the included articles. Of the included studies, 1305 duplicate studies were excluded. The remaining 1760 articles were screened based on the title and abstract and assessed for eligibility. The full text of 169 potentially eligible studies was evaluated by two independent authors (DK and $\mathrm{JH}$ ), of which 153 studies did not meet the inclusion criteria (reasons for exclusion are summarised in online supplemental figure 1). Sixteen studies, ${ }^{11-16} 31-40$ including 1127 postmenopausal women, met the inclusion criteria. Table 1 lists the summary and characteristics of each study.

Studies were published as early as 1989, and the follow-up duration after thyroidectomy ranged from 1 to 15 years. Three studies were prospective, ${ }^{131638}$ while three involved consecutive sampling (table 2). ${ }^{1433} 37$

All studies, except one, ${ }^{14}$ controlled for confounders in the study design using matching factors and exclusion criteria (table 2 and online supplemental table 2). The quality scores are shown in online supplemental table 3 , with scores ranging from 6 to 9 . All studies were found to be of moderate to high quality. After thyroidectomy, the mean TSH levels ranged between 0.00 and $1.8 \mathrm{mIU} / \mathrm{L}$. The postoperative levothyroxine doses are summarised in online supplemental table 2.

\section{Effects of TSH suppression on the lumbar spine}

All the included studies reported BMD of the lumbar spine in 426 postmenopausal women that underwent thyroidectomy for thyroid cancer and 701 controls. Overall, TSH suppression after thyroidectomy showed detrimental effects on the BMD of the lumbar spine (SMD $-0.46 ; 95 \%$ CI -0.77 to $-0.16 ; \mathrm{I}^{2}=77.6 \%$; $\left.=0.003\right)$. When analysed according to the TSH suppression target, stringent suppression, but not moderate suppression, showed negative effects on the postoperative BMD (SMD -0.55;
$95 \%$ CI -0.99 to $-0.10 ; \mathrm{I}^{2}=75.8 \% ; \mathrm{p}=0.016$ ) (figure $1 \mathrm{~A}$ ). Funnel plots indicated that there was no significant publication bias with stringent TSH suppression (TSH $<0.1 \mathrm{mIU} / \mathrm{L}$ ) (online supplemental figure 2A), which was supported by Egger's test $(\mathrm{p}=0.940)$.

\section{Effects of TSH suppression on the femoral neck}

Ten of the studies (282 postmenopausal women and 452 controls) investigated the effects of TSH suppression after thyroidectomy for thyroid cancer on the BMD of the femoral neck. ${ }^{11-1315163233363839}$ The BMD of the femoral neck was similar in TSH-suppressed postmenopausal women that underwent thyroidectomy for thyroid cancer compared with that in controls (SMD -0.32; 95\% CI -0.74 to $0.10 ; \mathrm{I}^{2}=81.8 \% ; \mathrm{p}=0.133$; figure $1 \mathrm{~B}$ ). This observation was similar across subgroups according to the suppressed TSH levels. Funnel plots indicated no significant publication bias (online supplemental figure 2B), as supported by Egger's test $(\mathrm{p}=0.177)$.

\section{Subgroup analyses}

A subgroup analysis showed that the association between stringent TSH suppression and lower BMD was consistent among studies with $>10$ years of follow-up (figure 2A). No significant differences in subgroups with different ethnicities and BMIs were detected. Furthermore, the detrimental effects of TSH suppression were more pronounced in patients who underwent total thyroidectomy than in those that underwent near-total thyroidectomy without statistical significance $(p=0.065)$. A significant interaction between surgical extent and BMD of the femoral neck (figure 2B; p for interaction=0.004) was observed. Moreover, total thyroidectomy was related to detrimental effects on the BMD of the femoral neck (SMD -0.60 ; $95 \%$ CI -0.89 to $-0.31 ; \mathrm{I}^{2}=90.4 \%$; $<<0.001$ ), but near-total thyroidectomy was not (SMD $0.00 ; 95 \%$ CI -0.30 to 0.30 ; $\left.\mathrm{I}^{2}=55.6 \% ; \mathrm{p}=0.996\right)$. The follow-up duration, ethnicity and BMI did not affect the association between TSH suppression and femoral neck BMD in postmenopausal patients who underwent thyroidectomy for thyroid cancer.

\section{DISCUSSION}

\section{Summary of the findings}

The potential effect of hyperthyroidism on postoperative bone health in patients who underwent thyroidectomy for thyroid cancer remains a concern in terms of longterm care. Identifying the groups that are at risk of deteriorating bone health after thyroidectomy is crucial for tailoring TSH suppression and guiding clinical treatment in a more focused and individualised direction. In this meta-analysis of 16 studies, including 1127 participants, we identified stringent TSH suppression after thyroidectomy for thyroid cancer that adversely affects the postoperative BMD in postmenopausal women. BMD is widely used for osteoporosis screening, and BMD changes are an independent risk factor for fracture. ${ }^{41}$ Our findings suggest that the oncological benefits of stringent 


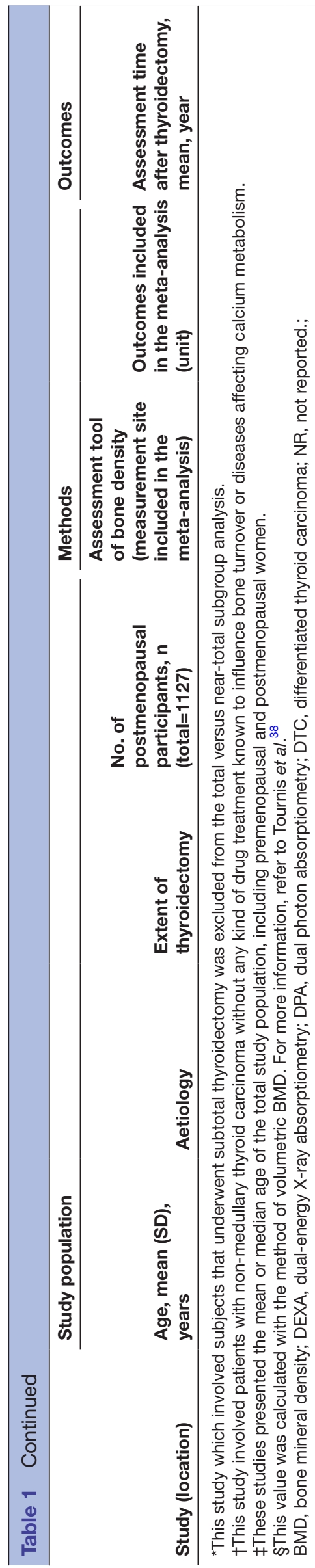

TSH suppression in postmenopausal women should be weighed against its possible adverse effects on postoperative bone health.

\section{Comparison with other studies}

Clinical guidelines recommend the administration of levothyroxine as the optimal treatment for patients undergoing thyroidectomy for thyroid cancer. Patients with residual carcinoma or those at a high risk of cancer recurrence, those at an intermediate risk of cancer recurrence and those at a low risk of cancer recurrence should maintain TSH levels at $<0.1,0.1-0.5$ and $0.5-2$ $\mathrm{mIU} / \mathrm{L}$, respectively. ${ }^{1718}$ Due to its beneficial effects on cancer recurrence, ${ }^{42}$ and overall survival, ${ }^{43}$ stringent TSH suppression is recommended for patients at a high risk of cancer recurrence. However, there is no consensus regarding serum TSH levels below the lower limit of the normal range. ${ }^{44}$ Because of the favourable prognosis associated with TSH suppression, patients with thyroid cancer receive life-long thyroxine treatment and have suppressed TSH levels for extended periods. Some studies have reported that long-term thyroxine therapy leads to an increased risk of bone fractures. ${ }^{45}{ }^{46}$ However, the effects of TSH suppression on the bone health of patients undergoing thyroidectomy for thyroid cancer are rarely discussed in the current guidelines, and few studies evaluate the adverse effects according to stratified TSH levels. Several recent population-based studies have reported associations between levothyroxine doses and bone health. ${ }^{1947}$ One study concluded that among patients with thyroid cancer, those who received levothyroxine had an increased risk of osteoporosis in a cumulative dose-dependent manner compared with those who did not receive levothyroxine (HR at a cumulative levothyroxine dose $>395 \mathrm{mg}$, 3.62; $95 \%$ CI 2.16 to 6.06 ; $\mathrm{HR}$ at a cumulative levothyroxine dose $\leq 265 \mathrm{mg}, 1.53$; $95 \%$ CI 0.91 to 2.57$).{ }^{47}$ In another study, 185956 patients with thyroid cancer that were administered a levothyroxine dosage of $\geq 170 \mu \mathrm{g} /$ day had a higher risk of osteoporotic fractures (HR 1.25; 95\% CI 1.07 to 1.45 ) than matched controls without cancer. ${ }^{19}$ However, as the intensity of TSH suppression therapy was stratified based on prescribed levothyroxine doses, results from these studies reflect the indirect effects of TSH concentration on the bone.

According to a series of previous studies, TSH suppression after thyroidectomy significantly reduced bone mass in postmenopausal women, whereas it did not affect premenopausal women and men. ${ }^{21} 248$ Menopause and the subsequent decrease in oestrogen are well-known risk factors that contribute to osteoporosis. ${ }^{49}$ The prominent effects of TSH suppression on bone health in postmenopausal women are clinically significant, and affected subjects need to be appropriately managed. We observed deleterious effects of stringent TSH suppression on the BMD of the lumbar spine. However, moderate TSH suppression did not significantly affect postoperative BMD. Inhibition of the receptor activator of nuclear 


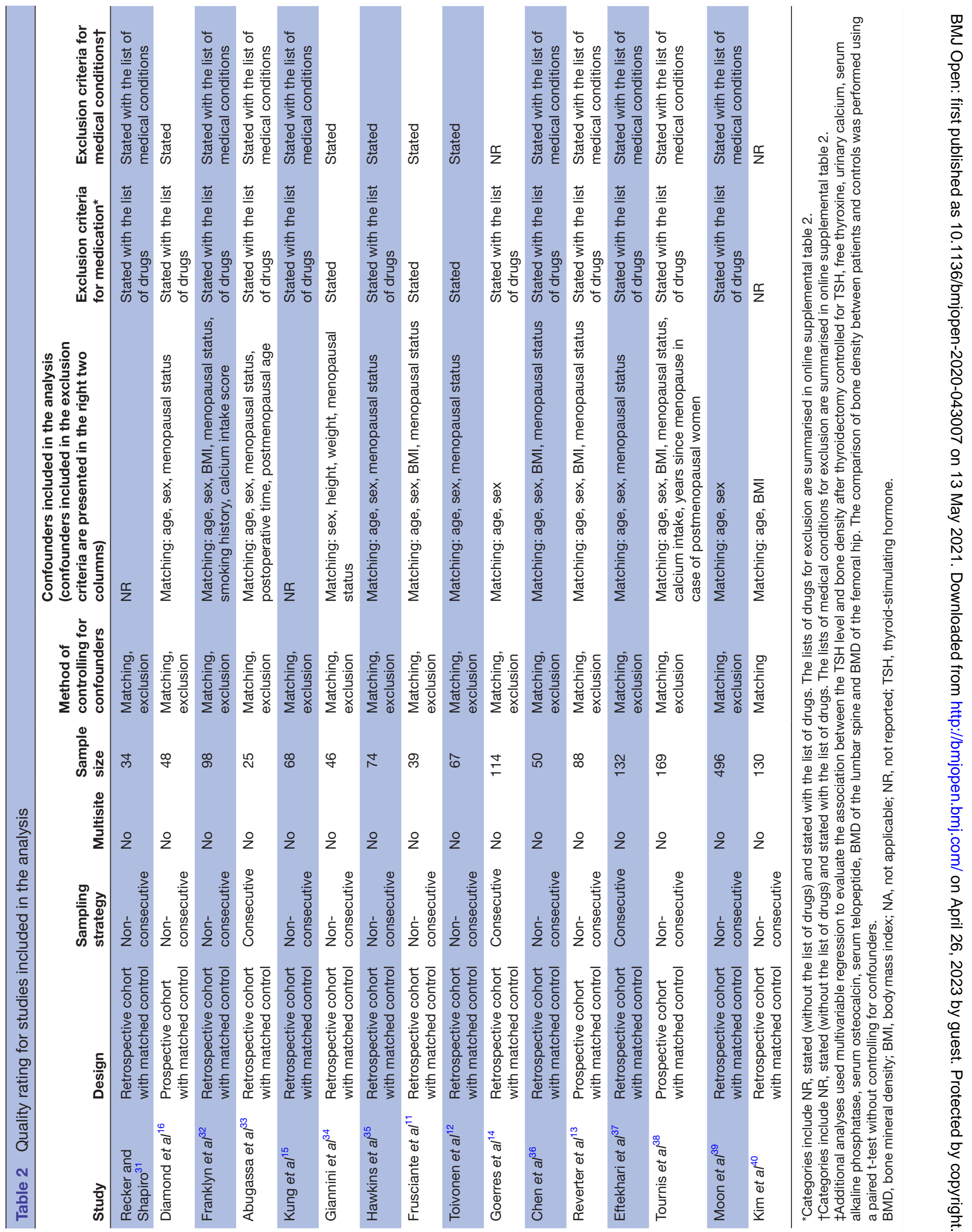




\section{A. Lumbar spine}

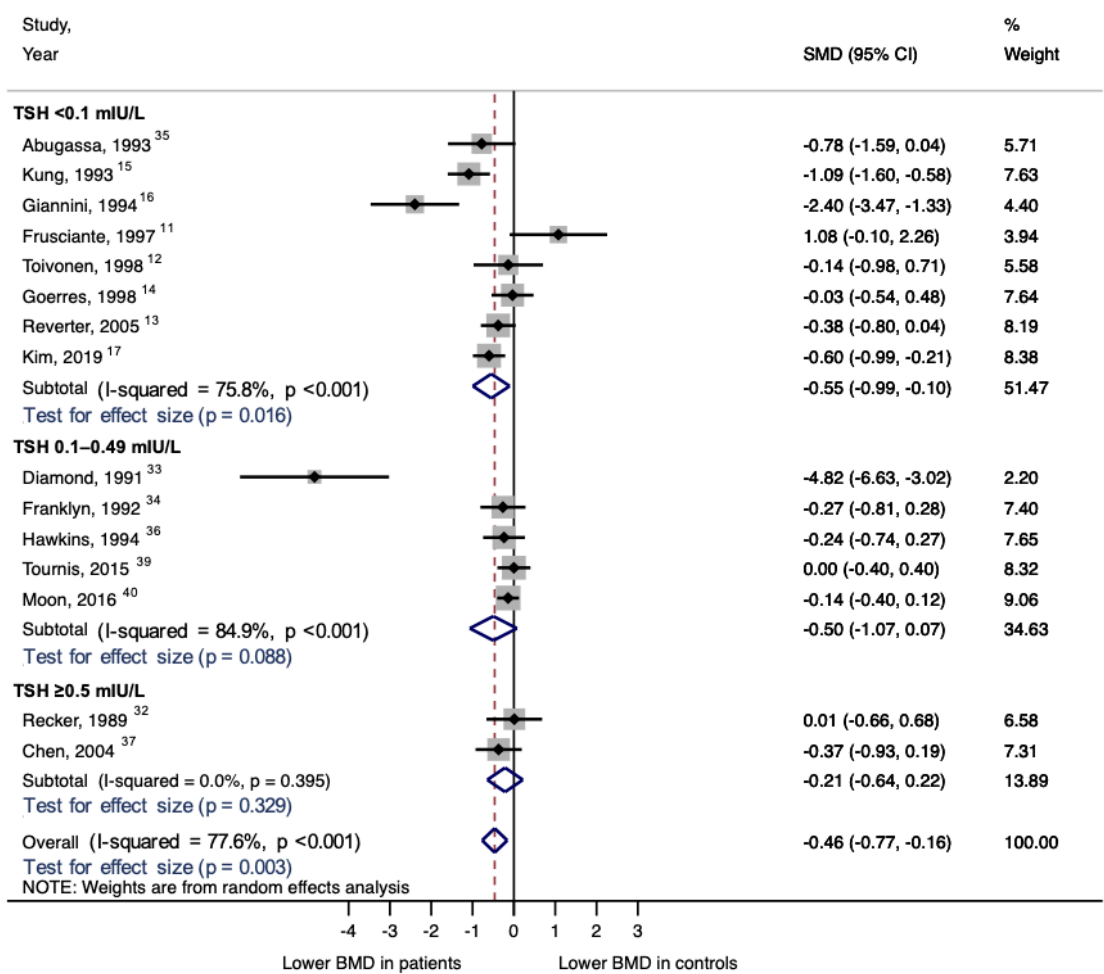

\section{B. Femoral neck}

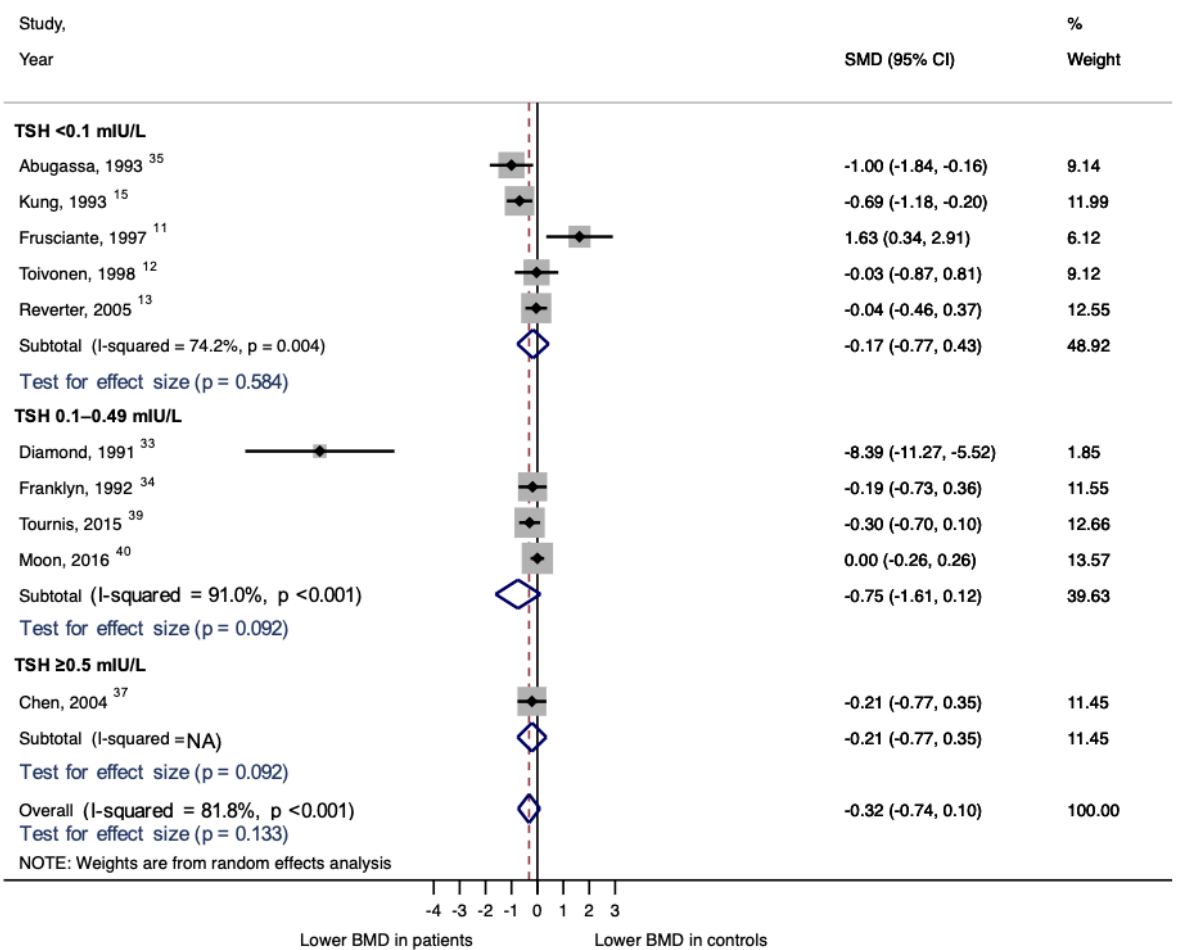

Figure 1 Postoperative BMD of lumbar spine (A) and femoral neck (B) in postmenopausal women undergoing TSH suppression after thyroidectomy for thyroid cancer. Meta-analysis results of case-control studies included in this review were presented according to TSH level. SMDs were calculated such that positive values indicated benefit for BMD in thyroidectomised group compared with controls without thyroid cancer. BMD, bone mineral density; NA, not available; SMD, standardised mean difference; TSH, thyroid-stimulating hormone. 


\section{A. Lumbar spine}

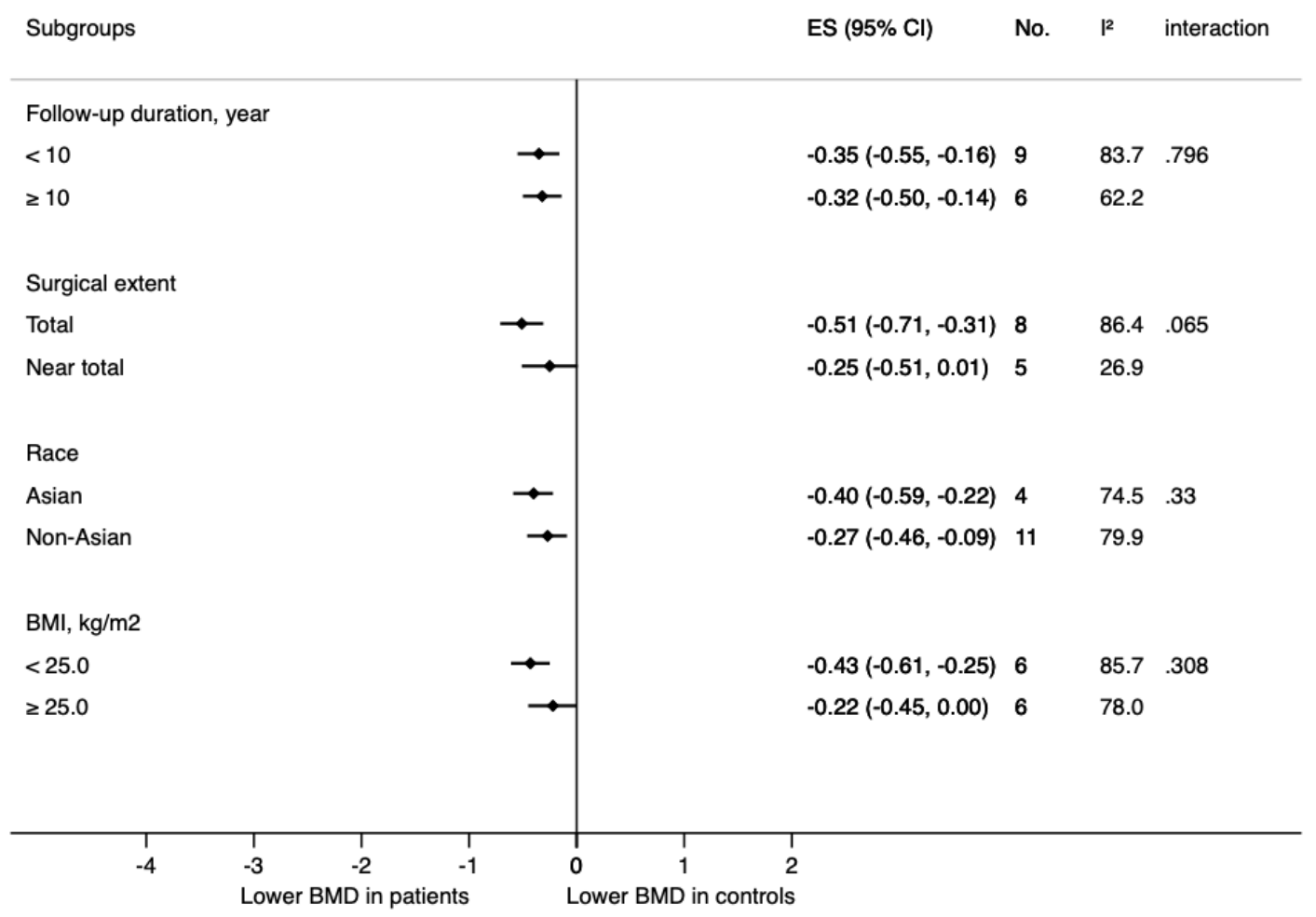

\section{B. Femoral neck}

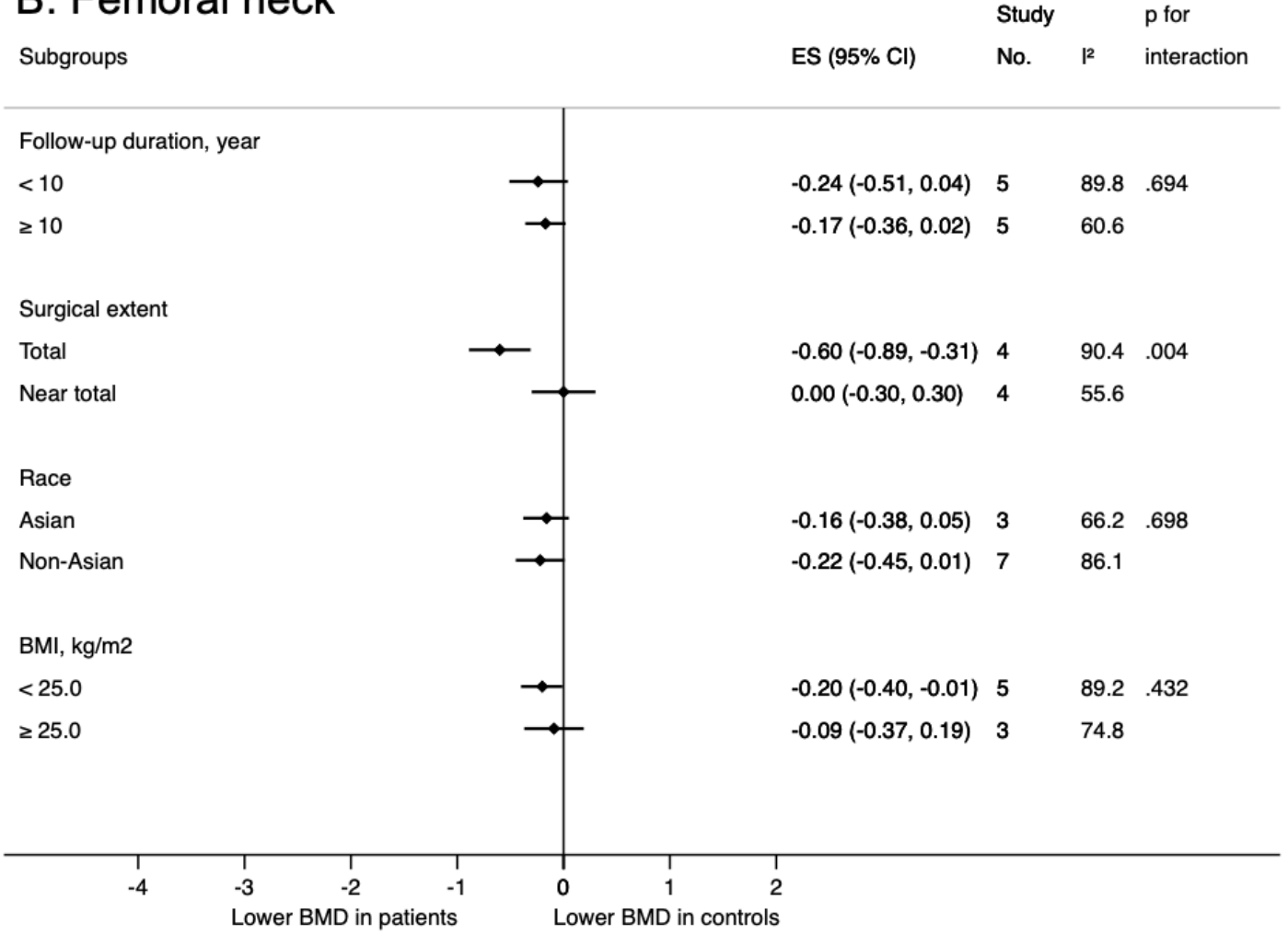

Figure 2 Subgroup analysis. Postoperative BMD of lumbar spine (A) and femoral neck (B) in postmenopausal women after thyroidectomy for thyroid cancer and subsequent TSH suppression by subgroups. BMD, bone mineral density; BMI, body mass index; ES, effect size; TSH, thyroid-stimulating hormone. 
factor- $\mathrm{\kappa B}$ ligand-induced osteoclast formation, ${ }^{50}$ and activation of Wnt-5a-mediated and $\beta$-arrestin 1-mediated osteoblast differentiation support that lower TSH levels may be responsible for lowering BMD. ${ }^{48}$

\section{BMD comparison on the lumbar spine and femoral neck}

The detrimental effects of TSH suppression have been reported to be more prominent on the BMD of the lumbar spine than on that of the femoral neck (figure 1). This result is consistent with that of previous studies. First, the lumbar spine is predominantly a trabecular bone. Its microarchitecture is more vulnerable to osteoporotic changes than that of the cortical bone, which comprises a large portion of the femoral neck. ${ }^{51}$ Second, the mineral contents of the lumbar spine change primarily during menopause, while those of the femoral neck change at a constant rate over one's lifetime. ${ }^{52}$ These factors may affect the interaction between the BMD of the lumbar spine and femoral neck and TSH and oestrogen levels in postmenopausal women. Low BMD of the lumbar spine is clinically significant as the spine is the most common osteoporotic fracture site, ${ }^{53}$ and spinal fractures are related to increased mortality. ${ }^{54} \mathrm{~A}$ recent population-based study assessing the risk of fracture after thyroidectomy by tracing patients for 40 years also reported a significant increase in the risk of vertebral fractures (standardised incidence ratio $2.8 ; 95 \%$ CI 2.3 to 3.3 ) and a relatively small increase in the risk of proximal femur fractures (standardised incidence ratio $1.3 ; 95 \%$ CI 1.0 to 1.8 ) after thyroidectomy. ${ }^{55}$

The deleterious effects of TSH suppression on the BMD of the lumbar spine were more prominent in patients who underwent total (vs near-total) thyroidectomy, in Asian (vs non-Asian) populations and in those with a $\mathrm{BMI}<25 \mathrm{~kg} /$ $\mathrm{m}^{2}$ (vs BMI $\geq 25 \mathrm{~kg} / \mathrm{m}^{2}$ ). Near-total thyroidectomy, which leaves a small amount of thyroid tissue $(<1.0 \mathrm{~mL})$, can be performed instead of total thyroidectomy, whereas partial or subtotal thyroidectomy is not appropriate for thyroid malignancy. ${ }^{1}$ Limited information is available regarding the differential effects of total and near-total thyroidectomy on bone health and BMD, suggesting the need for further research regarding this aspect. A study found that patients with a lower BMI that underwent thyroidectomy because of benign pathology required a significantly higher levothyroxine dose per kilogram to achieve a euthyroid state compared with those with a higher BMI (BMI $<25 \mathrm{~kg} / \mathrm{m}^{2}, 1.84 \pm 0.07 \mu \mathrm{g} / \mathrm{kg} /$ day; BMI $\geq 35 \mathrm{~kg} / \mathrm{m}^{2}$, $1.39 \pm 0.05 \mu \mathrm{g} / \mathrm{kg} /$ day); this association was age independent. ${ }^{56}$ We suggest levothyroxine dose adjustment and thorough monitoring of the BMD in patients without obesity undergoing thyroidectomy for thyroid cancer, followed by TSH suppression.

\section{Limitations}

Our study has several limitations. First, most of the included studies had a retrospective design, and the number of controlled confounding factors was heterogeneous in each study. Furthermore, studies may have been misclassified because of various factors known to affect TSH levels, such as factors related to the postmenopausal state, ${ }^{57}$ and parathyroid hormone levels ${ }^{58}$ Second, most of the included studies that involved TSH suppression reported the TSH level at a single time point. Although each study periodically assessed TSH levels to confirm maintenance within the intended level, fluctuations in TSH levels cannot be ruled out, and consequently, the effects of these fluctuations on BMD may represent potential confounders. Third, few studies have considered genetic bone susceptibility or the effects of lifestyle factors such as physical activity, smoking and alcohol consumption on the BMD. Fourth, some studies may have been misclassified because of the use of a first-generation TSH assay with insufficient sensitivity. ${ }^{59}$ Fifth, most of the cases were differentiated thyroid carcinoma, but thyroid cancer aetiology was not reported, or patients with other aetiologies were included. Therefore, additional treatments or differences in molecular pathogenesis may be the cause of heterogeneity. Sixth, most studies did not clarify whether all of the included patients were high risk or suppressed below the TSH level of $0.1 \mathrm{mIU} / \mathrm{L}$. Therefore, statistical analyses were performed based on the average TSH levels presented in each study. Seventh, other variables that may influence hypoparathyroidism or vitamin D levels, such as autoimmune conditions, genetic variants, radiation, mineral deposition and magnesium deficiency, were not evaluated. ${ }^{60}$ Eighth, only case-control studies were enrolled. A prospective cohort and longitudinal study are required. Ninth, funnel plots of the lumbar spine indicated that there was some publication bias with overall and moderate TSH suppression (TSH 0.10-0.49 $\mathrm{mIU} / \mathrm{L}$ ) (online supplemental figure 2A), as shown by the Egger's test ( $\mathrm{p}=0.098$ and 0.082 , respectively). Interpretation of the overall and moderate TSH suppression groups requires further investigation. Tenth, few studies have analysed the osteoporosis and fracture risk results according to the TSH suppression level, so this was not covered in our systematic review. Further investigation is needed regarding the benefits of TSH suppression therapy in relation to cancer recurrence or progression and the risk of osteoporosis or fractures, particularly in patients at a high risk of cancer recurrence receiving stringent TSH suppression therapy.

\section{CONCLUSIONS}

Stringent TSH suppression (TSH level $<0.10 \mathrm{mIU} / \mathrm{L}$ ) after thyroidectomy for thyroid cancer was found to exert detrimental effects on the postoperative BMD of the lumbar spine in postmenopausal women. Further welldesigned prospective studies are needed to evaluate the change in the BMD of different bones and the risk of bone fractures according to the TSH suppression levels in postmenopausal women who have undergone thyroidectomy for thyroid cancer to establish adequate postoperative management. 
Author affiliations

${ }^{1}$ Department of Orthopedic Surgery, Korea University College of Medicine, Seoul, The Republic of Korea

${ }^{2}$ Department of Orthopedic Surgery, 10th Fighter Wing Aeromedical Squadron, Suwon, The Republic of Korea

${ }^{3}$ Department of Medicine, Korea University College of Medicine, Seoul, The Republic of Korea

${ }^{4}$ Center for Obesity and Metabolic Diseases, Korea University Anam Hospital, Seoul, The Republic of Korea

${ }^{5}$ Department of Radiology, Spine Love Hospital, Goyang, The Republic of Korea ${ }^{6}$ Division of Foregut Surgery, Korea University College of Medicine, Seoul, The Republic of Korea

Acknowledgements The authors gratefully acknowledge Eun Ju Lee (Manager, Korea University Medical Library) for her valuable advice regarding the literature search for this article. The authors also thank the members of the Center for Obesity and Metabolic Diseases (Korea University Anam Hospital, Seoul, South Korea) for their administrative work.

Contributors DK designed the study, collected, extracted, analysed the data and wrote the manuscript. $\mathrm{JH}$ extracted and analysed the data and wrote the manuscript. YW interpreted the results and critically revised the manuscript. YK designed the study, collected the data and wrote the manuscript. SP supervised the study and critically revised the manuscript.

Funding This work was supported by grants from the Basic Science Research Program through the National Research Foundation of Korea funded by the Ministry of Education ((2020R111A1A01070106) (for Y.K.), and the Korea Medical Device Development Fund grant funded by the Korea government (the Ministry of Science and ICT, the Ministry of Trade, Industry and Energy, the Ministry of Health \& Welfare, the Ministry of Food and Drug Safety) (Project Number: 9991007295 , KMDF_PR_202012D13-02) (for S.P.)

Competing interests None declared.

Patient consent for publication Not required.

Ethics approval This study was approved by the Institutional Review Board of Korea University Anam Hospital (registration number: 2021AN0165).

Provenance and peer review Not commissioned; externally peer reviewed.

Data availability statement Data are available on reasonable request. Data are available on request. The data are available on request from the corresponding author (kukwon@korea.ac.kr).

Supplemental material This content has been supplied by the author(s). It has not been vetted by BMJ Publishing Group Limited (BMJ) and may not have been peer-reviewed. Any opinions or recommendations discussed are solely those of the author(s) and are not endorsed by BMJ. BMJ disclaims all liability and responsibility arising from any reliance placed on the content. Where the content includes any translated material, BMJ does not warrant the accuracy and reliability of the translations (including but not limited to local regulations, clinical guidelines, terminology, drug names and drug dosages), and is not responsible for any error and/or omissions arising from translation and adaptation or otherwise.

Open access This is an open access article distributed in accordance with the Creative Commons Attribution Non Commercial (CC BY-NC 4.0) license, which permits others to distribute, remix, adapt, build upon this work non-commercially, and license their derivative works on different terms, provided the original work is properly cited, appropriate credit is given, any changes made indicated, and the use is non-commercial. See: http://creativecommons.org/licenses/by-nc/4.0/.

\section{ORCID iD}

Jane Ha http://orcid.org/0000-0002-6175-8793

\section{REFERENCES}

1 Haugen BR, Alexander EK, Bible KC, et al. 2015 American thyroid association management guidelines for adult patients with thyroid nodules and differentiated thyroid cancer: the American thyroid association guidelines task force on thyroid nodules and differentiated thyroid cancer. Thyroid 2016;26:1-133.

2 Sugitani I, Fujimoto Y. Effect of postoperative thyrotropin suppressive therapy on bone mineral density in patients with papillary thyroid carcinoma: a prospective controlled study. Surgery 2011;150:1250-7.
3 Wang LY, Smith AW, Palmer FL, et al. Thyrotropin suppression increases the risk of osteoporosis without decreasing recurrence in Ata low- and intermediate-risk patients with differentiated thyroid carcinoma. Thyroid 2015;25:300-7.

$4 \mathrm{Lim} \mathrm{H}$, Devesa SS, Sosa JA, et al. Trends in thyroid cancer incidence and mortality in the United States, 1974-2013. JAMA 2017;317:1338-48.

5 Mazzaferri EL, Kloos RT. Clinical review 128: current approaches to primary therapy for papillary and follicular thyroid cancer. $J$ Clin Endocrinol Metab 2001:86:1447-63.

6 Biondi B, Filetti S, Schlumberger M. Thyroid-Hormone therapy and thyroid cancer: a reassessment. Nat Clin Pract Endocrinol Metab 2005;1:32-40.

7 Jódar E, Begoña López M, García L, et al. Bone changes in preand postmenopausal women with thyroid cancer on levothyroxine therapy: evolution of axial and appendicular bone mass. Osteoporos Int 1998:8:311-6.

8 Blum MR, Bauer DC, Collet T-H, et al. Subclinical thyroid dysfunction and fracture risk. JAMA 2015;313:2055-65.

9 Vestergaard P, Mosekilde L. Hyperthyroidism, bone mineral, and fracture risk-a meta-analysis. Thyroid 2003;13:585-93.

10 Nicholls JJ, Brassill MJ, Williams GR, et al. The skeletal consequences of thyrotoxicosis. J Endocrinol 2012;213:209-21.

11 Frusciante V, Carnevale V, Scillitani A, et al. Global skeletal uptake of technetium-99m methylene diphosphonate in female patients receiving suppressive doses of L-thyroxine for differentiated thyroid cancer. Eur J Nucl Med Mol Imaging 1998;25:139-43.

12 Toivonen J, Tahtela R, Laitinen K, et al. Markers of bone turnover in patients with differentiated thyroid cancer with and following withdrawal of thyroxine suppressive therapy. Eur $J$ Endocrinol 1998:138:667-73.

13 Reverter JL, Holgado S, Alonso N, et al. Lack of deleterious effect on bone mineral density of long-term thyroxine suppressive therapy for differentiated thyroid carcinoma. Endocr Relat Cancer 2005;12:973-81.

14 Goerres G, Theiler R, Müller-Brand J. Interfemur variation of bone mineral density in patients receiving high-dose thyroxin therapy. Calcif Tissue Int 1998;63:98-101.

15 Kung AWC, Lorentz T, Tam SCF. Thyroxine suppressive therapy decreases bone mineral density in post-menopausal women. Clin Endocrinol 1993;39:535-40.

16 Diamond T, Nery L, Hales I. A therapeutic dilemma: suppressive doses of thyroxine significantly reduce bone mineral measurements in both premenopausal and postmenopausal women with thyroid carcinoma. J Clin Endocrinol Metab 1991;72:1184-8.

17 Tuttle RM, Haddad RI, Ball DW, et al. Thyroid carcinoma, version 2.2014. J Natl Compr Canc Netw 2014;12:1671-80.

18 Perros P, Boelaert K, Colley S, et al. Guidelines for the management of thyroid cancer. Clin Endocrinol 2014;81:1-122.

19 Shin DW, Suh B, Lim H, et al. J-shaped association between postoperative levothyroxine dosage and fracture risk in thyroid cancer patients: a retrospective cohort study. J Bone Miner Res 2018;33:1037-43.

20 Bauer DC, Ettinger B, Nevitt MC, et al. Risk for fracture in women with low serum levels of thyroid-stimulating hormone. Ann Intern Med 2001;134:561-8.

21 Heemstra KA, Hamdy NAT, Romijn JA, et al. The effects of thyrotropin-suppressive therapy on bone metabolism in patients with well-differentiated thyroid carcinoma. Thyroid 2006;16:583-91.

22 Parker WAE, Edafe O, Balasubramanian SP. Long-Term treatmentrelated morbidity in differentiated thyroid cancer: a systematic review of the literature. Pragmat Obs Res 2017;8:57-67.

23 Quan ML, Pasieka JL, Rorstad O. Bone mineral density in welldifferentiated thyroid cancer patients treated with suppressive thyroxine: a systematic overview of the literature. J Surg Oncol 2002;79:62-70.

24 Yoon B-H, Lee Y, Oh HJ, et al. Influence of thyroid-stimulating hormone suppression therapy on bone mineral density in patients with differentiated thyroid cancer: a meta-analysis. J Bone Metab 2019;26:51-60.

25 Uzzan B, Campos J, Cucherat M, et al. Effects on bone mass of long term treatment with thyroid hormones: a meta-analysis. J Clin Endocrinol Metab 1996;81:4278-89.

26 Wang M-Y, Han Z-Q, Gong X-W, et al. TSH-suppressive therapy can reduce bone mineral density in patients with differentiated thyroid carcinoma: a meta-analysis. Eur Rev Med Pharmacol Sci 2020;24:922-9.

27 Moher D, Liberati A, Tetzlaff J, et al. Preferred reporting items for systematic reviews and meta-analyses: the PRISMA statement. Ann Intern Med 2009;151:264-9. 
28 Higgins JPT, Green S, Cochrane Collaboration. Cochrane Handbook for systematic reviews of interventions. Chichester: Wiley-Blackwell, 2009.

29 Wells GA, Shea B, O'Connell D. The Newcastle-Ottawa scale (NOS) for assessing the quality of Nonrandomised studies in meta-analyses. Ottawa, ON: Ottawa Hospital Research Institute, 2011. http://www. ohri.ca/programs/clinical_epidemiology/oxford.asp

30 Puhan MA, Schünemann HJ, Murad MH, et al. A grade Working group approach for rating the quality of treatment effect estimates from network meta-analysis. BMJ 2014;349:g5630.

31 Recker DP, Shapiro B. The effect of thyroidectomy on bone mineral content in perimenopausal women. Thyroidol 1989;1:59-65.

32 Franklyn JA, Betteridge J, Daykin J, et al. Long-Term thyroxine treatment and bone mineral density. Lancet 1992;340:9-13.

33 Abugassa S, Nordenström J, Eriksson S, et al. Bone mineral density in patients with chronic hypoparathyroidism. J Clin Endocrinol Metab 1993;76:1617-21.

34 Giannini S, Nobile M, Sartori L, et al. Bone density and mineral metabolism in thyroidectomized patients treated with long-term Lthyroxine. Clin Sci 1994;87:593-7.

35 Hawkins F, Rigopoulou D, Papapietro K, et al. Spinal bone mass after long-term treatment with L-thyroxine in postmenopausal women with thyroid cancer and chronic lymphocytic thyroiditis. Calcif Tissue Int 1994;54:16-19.

36 Chen C-H, Chen J-F, Yang B-Y, et al. Bone mineral density in women receiving thyroxine suppressive therapy for differentiated thyroid carcinoma. J Formos Med Assoc 2004;103:442-7.

37 Eftekhari M, Asadollahi A, Beiki D, et al. The long term effect of levothyroxine on bone mineral density in patients with well differentiated thyroid carcinoma after treatment. Hell J Nucl Med 2008;11:160-3.

38 Tournis S, Antoniou JD, Liakou CG, et al. Volumetric bone mineral density and bone geometry assessed by peripheral quantitative computed tomography in women with differentiated thyroid cancer under TSH suppression. Clin Endocrinol 2015;82:197-204.

39 Moon JH, Jung KY, Kim KM, et al. The effect of thyroid stimulating hormone suppressive therapy on bone geometry in the hip area of patients with differentiated thyroid carcinoma. Bone 2016;83:104-10.

$40 \mathrm{Kim} \mathrm{EH}$, Jeon YK, Pak K, et al. Effects of thyrotropin suppression on bone health in menopausal women with total thyroidectomy. $J$ Bone Metab 2019;26:31-8.

41 Berger C, Langsetmo L, Joseph L, et al. Association between change in BMD and fragility fracture in women and men. $J$ Bone Miner Res 2009;24:361-70.

42 Cooper DS, Specker B, Ho M, et al. Thyrotropin suppression and disease progression in patients with differentiated thyroid cancer: results from the National thyroid cancer treatment cooperative registry. Thyroid 1998;8:737-44.

43 Jonklaas J, Sarlis NJ, Litofsky D, et al. Outcomes of patients with differentiated thyroid carcinoma following initial therapy. Thyroid 2006;16:1229-42.
44 Biondi B, Cooper DS. Benefits of thyrotropin suppression versus the risks of adverse effects in differentiated thyroid cancer. Thyroid 2010;20:135-46.

45 Flynn RW, Bonellie SR, Jung RT, et al. Serum thyroid-stimulating hormone concentration and morbidity from cardiovascular disease and fractures in patients on long-term thyroxine therapy. J Clin Endocrinol Metab 2010;95:186-93.

46 Viniol A, Hickstein L, Walker J, et al. Influence of thyroid hormone therapy on the fracture rate - a claims data cohort study. Bone 2016;86:86-90.

47 Lin S-Y, Lin C-L, Chen H-T, et al. Risk of osteoporosis in thyroid cancer patients using levothyroxine: a population-based study. Curr Med Res Opin 2018;34:805-12.

48 Faber J, Galløe AM. Changes in bone mass during prolonged subclinical hyperthyroidism due to L-thyroxine treatment: a metaanalysis. Eur J Endocrinol 1994;130:350-6.

49 Pacifici R, Estrogen PR. Estrogen, cytokines, and pathogenesis of postmenopausal osteoporosis. J Bone Miner Res 1996;11:1043-51.

50 Abe E, Marians RC, Yu W, et al. Tsh is a negative regulator of skeletal remodeling. Cell 2003;115:151-62.

51 Albright F, Smith PH, Richardson AM. Postmenopausal osteoporosis: its clinical features. JAMA 1941;116:2465-74.

52 Schaadt O, Bohr H. Different trends of age-related diminution of bone mineral content in the lumbar spine, femoral neck, and femoral shaft in women. Calcif Tissue Int 1988;42:71-6.

53 Ensrud KE. Epidemiology of fracture risk with advancing age. J Gerontol A Biol Sci Med Sci 2013;68:1236-42.

54 Ensrud KE, Thompson DE, Cauley JA, et al. Prevalent vertebral deformities predict mortality and hospitalization in older women with low bone mass. J Am Geriatr Soc 2000;48:241-9.

55 Melton LJ, Ardila E, Crowson CS, et al. Fractures following thyroidectomy in women: a population-based cohort study. Bone 2000;27:695-700.

56 Ojomo KA, Schneider DF, Reiher AE, et al. Using body mass index to predict optimal thyroid dosing after thyroidectomy. J Am Coll Surg 2013;216:454-60.

57 Ballinger CB, Browning MCK, Smith AHW. Hormone profiles and psychological symptoms in peri-menopausal women. Maturitas 1987;9:235-51.

58 Mosekilde L, Christensen MS. Decreased parathyroid function in hyperthyroidism: interrelationships between serum parathyroid hormone, calcium-phosphorus metabolism and thyroid function. Acta Endocrinol 1977;84:566-75.

59 Goichot B, Sapin Rémy, Schlienger JL. Subclinical hyperthyroidism: considerations in defining the lower limit of the thyrotropin reference interval. Clin Chem 2009;55:420-4.

60 Hakami Y, Khan A. Hypoparathyroidism. Front Horm Res 2019;51:109-26. 